\title{
Levodopa Induced Movement Disorders
}

\author{
Behzad Saberi* \\ Medical Research, Iran
}

*Corresponding author: Behzad Saberi, MD, Medical Research, Iran.

Received Date: April 30, 2019

Published Date: May 24, 2019

\section{Opinion}

In many patients under levodopa treatment, involuntary movements would eventually happen. Such dyskinesias are similar to tardive dyskinesias in appearance. Diphasic dyskinesias or dyskinesia-improved-dyskinesia (DID) can be seen during levodopa treatment, sometimes in combination with dystonia and usually appears with changes in dopamine levels. "Square-wave" dyskinesias and "Off" dystonia are rare patterns of dyskinesias. Dyskinesia-parkinsonism which would be dyskinesias in one part and parkinsonism in other part of the body, respiratory and ocular dyskinesias also can be seen in the patients.

Movement disorders induced by levodopa can appear in early phases of the disease but usually after years of treatment, when the disease goes into its moderate to severe phases and when the patient's benefit from treatment becomes lower which is so called motor fluctuations. Amount of medication which the patient takes, the duration of treatment and disease and its severity and the cause of the disease can determine the appearance of such movement disorders [1-5]. Hyperkinetic movement disorders caused by levodopa can be classified as: 1 . Typical forms of dyskinesia, 2 . Less usual forms of dyskinesia and 3. Other forms of hyperkinetic movements. Dyskinesia-parkinsonism, Diphasic dyskinesiadystonia, Peak-dose dystonia and dyskinesia and Off period dystonia are typical forms of dyskinesia. Ocular, respiratory and square wave dyskinesias are less usual forms of dyskinesia and Enhanced tremor and Akathisia are other hyperkinetic movements related to levodopa treatment. Ameliorating levodopa dyskinesias can be achieved by using dopamine agonists, adding amantadine, adding catechol-o-methyltrasferase (COMT) inhibitors like entacapone or tolcapone and reducing levodopa. Amantadine can cause psychosis and hallucinosis and clozapine may cause agranulocytosis which such side effects may reduce their clinical usage [5-9]. Deep brain stimulation (DBS) of the globus pallidus or subthalamic nucleus can be used to reduce dyskinesias. Worsening dementia, infection, stroke, lead fracture or erosion and ICH can be side effects of using such treatment method. Continuous supply of dopaminergic stimulation is another strategy to ameliorate dyskinesias. Delivering levodopa into the intestines and apomorphine into the bloodstream are examples of using such treatment method. Employing these treatment methods requires more studies and trials to be done more effectively in clinical practice.

\section{Acknowledgement}

None.

\section{Conflict of Interest}

No conflict of interest.

\section{References}

1. Burkhard PB (2014) Acute and subacute drug-induced movement disorders. Parkinsonism Rel Disord 20S1: S108-S112.

2. Sankar T, Lozano AM (2011) Surgical approach to L-DOPA-induced dyskinesias. Int Rev Neurobiol 98: 151-171.

3. Zesiewicz TA, Sullivan KL (2011) Drug-induced hyperkinetic movement disorders by non-neuroleptic agents. Handb Clin Neurol 100: 347-363.

4. Fisone G, Bezard E (2011) Molecular mechanisms of L-dopa induced dyskinesia. Int Rev Neurobiol 98: 95-122.

5. Prashanth LK, Fox S, Meissner WG (2011) L-dopa-induced dyskinesiaclinical presentation, genetics, and treatment. Int Rev Neurobiol 98: 3154 .

6. Politis M, Wu K, Loane C, Brooks DJ, Kiferle L, et al. (2014) Serotonergic mechanisms responsible for levodopa-induced dyskinesias in Parkinson's disease patients. J Clin Invest 124: 1340-1349.

7. Huot P, Johnston TH, Koprich JB, Fox SH, Brotchie JM (2013) The pharmacology of L-DOPA-induced dyskinesia in Parkinson's Disease. Pharmacol Rev 65: 171-222.

8. Jost WH (2014) Unwanted effects and interaction of intrajejunal levodopa/carbidopa administration. Expert Opin Drug Saf 13: 447-458.

9. Diederich NJ, Goetz CG (1998) Drug induced movement disorders. Neurol Clinics 16: 125-139. 